\title{
An Assessment of the RAIM Performance of a Combined Galileo/GPS Navigation System using the Marginally Detectable Errors (MDE) Algorithm
}

\author{
W. Y. Ochieng, K. F. Sheridan, K. Sauer and X. Han \\ Department of Civil and Environmental Engineering \\ Imperial College of Science, Technology and Medicine \\ London SW7 $2 B U$ \\ Tel: +44 (0) 2075946104 \\ Fax: +44 (0) 2075946102 \\ Email:w.ochieng@ic.ac.uk \\ P. A. Cross \\ (University College London) \\ S. Lannelongue, N. Ammour and K. Petit \\ (Alcatel Space)
}

\section{ABSTRACT}

Integrity relates to the trust that can be placed in the correctness of information supplied by a navigation system. It includes the ability of the navigation system to provide timely warnings to users when the system fails to meet its stated accuracy. Specifically, a navigation system is required to deliver a warning (alarm) when the error in the derived user position solution exceeds an allowable level (alarm limit). This warning must be issued to the user within a given period of time (time-to-alarm) and with a given probability (integrity risk). The two main approaches to monitoring the integrity of satellite navigation systems are Receiver Autonomous Integrity Monitoring (RAIM), and monitoring based on an independent network of integrity monitoring stations and a dedicated Ground Integrity Channel (GIC). More recently Satellite Autonomous Integrity Monitoring (SAIM) methods have also been investigated.

This paper presents the results of a study to assess the RAIM capability of the Galileo system when used alone and when combined with the Global Positioning System (GPS). The assessment was based on the Marginally Detectable Error (MDE) algorithm. The results show a significant improvement in the capability to perform RAIM using a combined Galileo/GPS system compared to the performance using the Galileo system alone. This study was supported by Alcatel Space and was a contribution to the Galileo definition studies carried out for the European Community under the GALA project. 


\section{INTRODUCTION}

The Galileo system currently under definition is the European contribution to the second generation of global navigation satellite systems (GNSS 2). It has been proposed as a global, satellite based navigation system and will support multi-modal transport navigation requirements and many other applications requiring spatial and/or temporal information, plus derivatives, to users equipped with suitable Galileo receivers. It has also been proposed that Galileo will be compatible and interoperable with GPS, GLONASS, space based augmentation systems (SBAS) and ground based augmentation systems (GBAS) currently under development. The system which is expected to achieve full operational capability (FOC) by the year 2008 will be under European civil control and open to international participation.

Of the navigation services identified during the definition studies, it is envisaged that only a subset of these services will be supported entirely by the Galileo system. The others could be supported through the combined use of the Galileo system with other sensors and other systems such as GPS. From the user perspective, and considering the current systems (GPS and GLONASS), and those under development (EGNOS, WAAS and MSAS), potential exists for enhanced navigation performance when data from other systems are combined with that from the Galileo system. For this reason, it is important to investigate the impact on the performance levels achievable and hence the potential user services that would benefit from such a combined approach.

\section{INTEGRITY MONITORING}

The required navigation performance (RNP) of a system is typically specified in terms of the four parameters of accuracy, integrity, continuity and availability (Ochieng et al., 1999).

(1) Accuracy is defined as the degree of conformance of an estimated or measured position at a given time, to the truth. 
(2) Integrity relates to the trust, which can be placed in the correctness of the information supplied by the navigation system. It includes the ability of the navigation system to provide timely warnings to users when the system must not be used for navigation/positioning. Specifically, a navigation system is required to deliver a warning (alarm) of any malfunction (an alarm limit being exceeded) to users within a given period of time (time-to-alarm) and with a given probability (integrity risk).

(3) Continuity is defined as the ability of the total system to perform its function without interruption during an intended period of operation. Continuity risk is the probability that the system will be interrupted and not provide guidance information for the intended period of operation. This risk is a measure of system unreliability.

(4) Availability is defined as the percentage of time during which the service is available for use, taking into account all outages, whatever their origins. The service is available if all the accuracy, integrity and continuity requirements are satisfied.

Of the RNP parameters given above, integrity is the one that relates most directly to safety and is therefore a crucial element, particularly for those applications that are safety-of-life (SOL) critical such as civil aviation. The different approaches to the monitoring of integrity of stand-alone and augmented satellite based navigation systems are:

- Receiver Autonomous Integrity Monitoring (RAIM).

- Ground based integrity monitoring using an independent network of integrity monitoring stations and a dedicated Ground Integrity Channel (GIC).

- Satellite Autonomous Integrity Monitoring (SAIM) based on the monitoring of the performance of the frequency generation mechanism on board the satellite.

These methods are complementary and for very strict SOL integrity requirements, more than one technique will usually be required (Fernow and Loh, 1994). 


\section{Receiver Autonomous Integrity Monitoring (RAIM)}

Receiver Autonomous Integrity Monitoring (RAIM) is a method applied by the user (i.e. the receiver) to check the consistency of the measurements made from different satellites to estimate the quality of the resulting position. RAIM methods therefore, require redundant measurements and good constellation geometry. Range measurements must be available from a minimum of five satellites, with adequate geometry, to allow an anomaly to be detected, a minimum of six satellites are required to remove the faulty satellite observations from the solution.

In order to carry out a RAIM capability analysis, the definition of integrity given above has to be transformed into quantifiable requirements. Performance thresholds must be specified in terms of alarm limits, integrity risk, time-to-alarm, false alarm rates and probability of missed detection. The constellation configuration and the corresponding User Equivalent Range Error (UERE) budgets must also be known. Additionally, the coverage area and spatial and temporal sampling interval must be defined.

The Galileo constellation used for the simulations carried out during this study was the Walker model of 30 medium Earth orbit (MEO) satellites, evenly distributed in three orbital planes (Vincent, 2000). The predicted GPS constellation for 2008-2010 was based on the current launch schedule and modernisation plans. Every GPS and Galileo satellite was assigned a predicted UERE budget based on previous analysis from the Galileo definition study and official United States Department of Transport/Department of Defence (DoT/DoD) estimates_(Lannelongue et al., 2000; Ochieng and Cross, 2000a, 2000b; Turner et al., 2000). Appropriate mapping functions have been used to model the effects of satellite elevation on the UERE. Table 1 presents UERE values for GPS and Galileo satellites at selected elevations. The results presented in this paper have been derived using the GALA estimates for GPS satellites, applying L1/L2 values to block IIR-modified satellites and L1/L5 values to block IIF satellites. The Galileo satellites were assigned the UERE budgets defined for CAS, Controlled Access Service, for professional market 
applications. Table 2 lists the integrity performance thresholds for Galileo used during this research (Ochieng and Cross, 2000a; 2000b). Further parameters are derived in the following section. The capability to perform RAIM depends on the thresholds specified.

\section{RAIM AND THE MDE ALGORITHM}

The integrity algorithm comprises two main steps. Firstly, the minimum size of gross error, sometimes referred to as a blunder or outlier, in an observation that will be detected at a given probability is determined, any larger errors will be detected, smaller errors will not. Secondly, the impact that the smallest detectable error would have on the estimated receiver position is quantified to determine if it leads to a positioning error exceeding the alarm limit.

\section{Computing the Marginally Detectable Error}

The Marginally Detectable Error $\left(\boldsymbol{M D E} \boldsymbol{E}_{\boldsymbol{i}}\right)$ for an observation, $\boldsymbol{i}$, is the minimum size of gross error that will be detected at a selected probability, and is given by (Cross, 1983),

$$
M D E_{i}=\delta_{i}^{u} P_{i} \quad(1)
$$

where,

$\delta_{i}^{u}$ depends only on the chosen probabilities, $\alpha$ and $\beta$ of Type 1 (i.e. the probability of false alarm) and Type 2 (the probability of missed detection) errors respectively, and is expressed as,

$$
\delta_{i}^{u}=a+b
$$

where,

$\boldsymbol{a}$ and $\boldsymbol{b}$ are determined using the probability of Type 1 and Type 2 errors respectively, and assuming a normal distribution,

and,

$$
\boldsymbol{P}_{i}=\frac{\sigma_{i}^{2}}{\sigma_{v_{i}}}
$$

where, 
$\sigma_{i}^{2}$ is the variance of observation $\boldsymbol{i}$, and

$\sigma_{v_{i}}$ is the standard deviation of the residual $\boldsymbol{v}_{i}$.

The variance for each observation $\boldsymbol{i}$ is the square of the UERE for satellite $\boldsymbol{i}$, and is contained on the leading diagonal of the covariance matrix of the observations, $\boldsymbol{C}_{\boldsymbol{l}}$. The standard deviation of the residual $\boldsymbol{i},\left(\sigma_{v_{i}}\right)$ is the square-root of the variance, from the leading diagonal of the covariance matrix of residuals $\boldsymbol{C}_{\boldsymbol{v}}$ which is given by,

$$
C_{v}=C_{l}-A C_{x} A^{T}
$$

where,

$C_{l} \quad$ is the covariance matrix of the observations,

$\boldsymbol{A}$ is the design matrix relating the observations to the estimated parameters, and,

$\boldsymbol{C}_{\boldsymbol{x}}$ is the covariance matrix of the estimated parameters, $\left[\boldsymbol{C}_{x}=\left(\boldsymbol{A}^{T} \boldsymbol{W} \boldsymbol{A}\right)^{-1}\right]$, where,

$\boldsymbol{W}$ is the weight matrix of the observations $\left[\boldsymbol{W}=\boldsymbol{C}_{l}^{-1}\right]$.

\section{Computing the Position Shift due to each MDE}

Having determined the minimum gross error that will be detected at the specified probability from each observation, the impact that this error has on the estimated position is now computed from,

$$
x=\left(A^{T} W A\right)^{-1} A^{T} W b
$$

where,

$\boldsymbol{x} \quad$ is the vector of estimated parameters, and,

$\boldsymbol{b} \quad$ is a null vector, except for the $i$ th element which is $\boldsymbol{M D E} \boldsymbol{E}_{\boldsymbol{i}}$

i.e. $\quad b^{T}=\left[0,0,0, M D E_{i}, 0,0,0,0,0\right]$

\section{CAPABILITY TO PERFORM RAIM}

The process of determining whether the capability to perform RAIM exists (i.e. RAIM availability) proceeds as follows; 
(1) Determine the MDE for each observation [equation 1]

(2) Compute the position shift due to the MDE from each satellite in turn [equation 5]

(3) Test the position shifts against the alarm limits.

(4) If the shifts are within the alarm limits then the capability to perform RAIM exists (i.e. RAIM is available).

When RAIM is available, if an outlier that would produce a position error in excess of the alarm limit occurred in an observed range, then it would be identified by the receiver's integrity algorithm at the selected probability level.

\section{Setting Probability Levels for the Study}

The integrity requirements for the Galileo user services have been specified in terms of integrity risk, vertical and horizontal alarm limits, and time-to-alarm. In order to carry out a RAIM capability analysis, the probabilities of missed detection and false alarm must also be specified.

Integrity risk is the probability during the period of operation that an error, whatever the source, will result in a computed position error exceeding the alarm limit, and that the user will not be informed within the specified time-to-alarm.

Risk $=$\begin{tabular}{|c|c|}
$\begin{array}{c}\text { Probability of a blunder occurring } \\
\text { that will cause a position error } \\
\text { exceeding the alarm limit }\end{array}$
\end{tabular}$*$\begin{tabular}{c}
$\begin{array}{c}\text { Probability of } \\
\text { missed detection }(\beta)\end{array}$ \\
\hline
\end{tabular}

The missed detection probability $(\beta)$ required to meet the integrity risk of $10^{-7} / \mathrm{hr}$ specified for the Galileo system can be determined if the probability of a blunder occurring within the system is known.

\section{Determination of the probability of a blunder occurring within the system}

At the time this study was conducted a value for the probability of a blunder occurring had not been specified for the Galileo system. As part of this research, a value was determined by 
assessing the unscheduled failure modes of the GPS satellites. This value has been used both for the GPS and Galileo satellites. The process adopted for the unscheduled satellite failure mode analysis is given below.

\section{Unscheduled Simultaneous GPS Satellite Failure Analysis}

The analysis of simultaneous unscheduled satellite failures has been carried out under three main assumptions (Phlong \& Elrod, 1994).

- All GPS satellites are identical, and operate identically and independently.

- The constellation is in a steady state, i.e. the satellites in orbit have randomly distributed ages.

- All failure probability density functions have exponential models of the form:

$$
f(t)=\lambda e^{-\lambda t}
$$

where, $\lambda={ }^{1} /{ }_{\text {MтвF }}$ (Mean Time Between Failure), with a corresponding life distribution of:

$$
F(t)=\int_{0}^{t} \lambda e^{-\lambda x} d x=\left[-e^{-\lambda x} J_{0}^{t}=1-e^{-\lambda t}\right.
$$

The corresponding Reliability function is therefore given by the expression:

$$
R(t)=1-F(t)=e^{-\lambda t}
$$

From the above expressions, the probability $\left(\boldsymbol{P}_{f}\right)$ that at time $\boldsymbol{t}_{\boldsymbol{\theta}}$, a satellite is working well and that during the period $\boldsymbol{t}_{\boldsymbol{0}}+\boldsymbol{k}$ (where $\boldsymbol{k}$ is the period of operation) a failure occurs is given by the following expression.

$$
\frac{F\left(t_{0}+k\right)-F\left(t_{0}\right)}{\boldsymbol{R}\left(t_{0}\right)}=\frac{e^{-\lambda t_{0}}-e^{-\lambda\left(t_{0}+k\right)}}{e^{-\lambda t_{0}}}=1-e^{-\lambda k}
$$

From expression (9) it can be seen that the probability of failure only depends on the parameter $\boldsymbol{k}$, i.e. the period of operation. It does not depend on $\boldsymbol{t}_{\boldsymbol{0}}$ (corresponding to lack of memory).

Given the number of satellites in the constellation $\boldsymbol{m}$, and the number of unscheduled simultaneous satellite failures $\boldsymbol{n}$, the probability that $\boldsymbol{n}$ satellites fail while the remaining $\boldsymbol{m}-\boldsymbol{n}$ satellites are operational is given by: 


$$
\boldsymbol{P}_{f_{n}}=\boldsymbol{C}_{m}^{n} \boldsymbol{P}_{f}^{n}\left(1-\boldsymbol{P}_{f}\right)^{m-n}
$$

The frequency of failures over a period of $\boldsymbol{T}$ years can then be obtained from,

$$
\boldsymbol{F}_{n}=\boldsymbol{P}_{f_{n}} \boldsymbol{T} \quad \text { (11) }
$$

Using the above expressions, the probability of any number of simultaneous satellite failures can be determined. For this study the calculations have been based on the GPS satellite design value for MTBF of 2346 hours, this is equivalent to 3.73 failures per satellite per year (Conley, 1998). An example of the output of this process for a 54 satellite constellation, i.e. Galileo plus GPS, used for subsequent analysis is a probability of failure of one satellite estimated at $2.2 \times 10^{-2}$ unscheduled failures per hour. Note that the instantaneous simultaneous failure of more than one satellite is highly unlikely (e.g. a probability of failure of $3 \times 10^{-8}$ for the two satellite case). In this case extended RAIM algorithms could be used.

\section{The probability of missed detection}

The missed detection probability $(\beta)$ needed to satisfy the risk criteria given this frequency of unscheduled satellite failures is $4.5 \times 10^{-6}$. The $\boldsymbol{b}$ value used in equation (2) is derived from a normal distribution using this probability figure (i.e. $b=4.433$ ).

\section{The probability of false alarm}

It is apparent from equation (2) that to compute the test statistic $\boldsymbol{\delta}_{\boldsymbol{i}}{ }_{i}, \boldsymbol{a}$ must also be derived. This is accomplished using a similar assumption of normal distribution and a probability $\alpha$, which is the probability of a false alarm. The false alarm rate must be set by the user for a particular application.

The RTCA MOPS false alarm rate for GPS WAAS airborne equipment is $1 \times 10^{-5} / \mathrm{hr}$ (RTCA, 1998). With the minimum time interval between independent samples set at $150 \mathrm{~s}$, the false alarm probability is equal to $4 \times 10^{-7}$, in line with previous Galileo studies this figure has been simplified to $1 \times 10^{-6}$. This independent sampling interval was determined during previous projects conducted 
by Alcatel Space Industries and assumes that the clock, ephemeris and ionospheric errors have been corrected. Alternative false alarm rates have been proposed for different applications, for example the RTCA MOPS figure for supplemental navigation is 0.002 alarms per hour (RTCA, 1991). Assuming the same time interval of 150 s for independent samples, the false alarm probability per sample is $8 \times 10^{-5}$. In the GPS risk assessment carried out by the John Hopkins University (Corrigan et al., 1999) the assumption is made that with the removal of selective availability there are only 10 independent measurements per hour, i.e. an interval of 360 s. Applying this figure to the hourly false alarm rates would clearly produce more alternative figures for false alarm probabilities.

As the values for the probability of missed detection $(\beta)$ and false alarm $(\alpha)$ will vary in practice, the simulation program has been run with two alternative scenarios (Table 3) that reflect the range of values from the sources described. Scenario 1 assumes relatively high probabilities for missed detection $(\beta)$ and false alarm $(\alpha)$, whilst scenario 2 imposes more stringent constraints.

The algorithms and input parameters have been implemented in GNSS design software developed at the London Centre for GNSS Research (LCGR).

\section{RESULTS AND ANALYSIS}

\section{RAIM Availability}

The capability to perform RAIM with Galileo only and a combined Galileo/GPS constellation has been assessed using the marginally detectable errors (MDE) algorithm. If RAIM is available then a receiver has enough information to be able to detect the presence of errors of a certain size at a certain probability. As the required probability levels will vary with the positioning application, scenarios have been tested using two alternative probability levels. The minimum probability level has been set at a relatively relaxed level, i.e. a relatively high proportion of errors will remain undetected. The maximum probability level reflects more stringent requirements, e.g. for 
safety of life applications, for which the probability of detecting an error must be considerably higher.

Using the two scenarios in Table 3 the proportion of time at which RAIM is available has been determined. Table 4 shows the percentage of samples, at a 5-minute time intervals and 5-degree ground resolution computed over 24 hours, at which the minimum size of position shift that could be detected was within the $18 \mathrm{~m}$ vertical and horizontal alarm limit, i.e. RAIM availability. The temporal and spatial sampling intervals are the same as those used in the GPS risk assessment carried out at the John Hopkins University (Corrigan et al., 1999). During this study a denser sampling grid and a 1-minute time interval were tested and found not to alter the results in any significant way. RAIM availability figures are given on a regional and global scale, as defined for the potential Galileo service coverage.

These figures show some important points: -

- The increased redundancy and improved constellation geometry in the outlier detection algorithm resulting from a combined constellation improves RAIM availability significantly. Using Galileo satellites alone, it is only possible to detect errors leading to vertical shifts greater than the $18 \mathrm{~m}$ alarm limit for $65 \%$ of the samples at the minimum probability level, at the maximum probability level this figure falls as low as $7 \%$. With the combined Galileo/GPS constellation RAIM is available almost $100 \%$ of the time at the minimum probability level and $92 \%$ of the time at the maximum probability level, a significant improvement. RAIM availability levels for regional and global coverage do not differ significantly.

- RAIM availability clearly depends on the specified performance parameters, including alarm limits and probabilities of missed detection and false alarm. Horizontal performance is significantly better than vertical. If horizontal and vertical alarm limits are set to the same value, as they were when this research was undertaken, then it is only the vertical component 
that will dictate whether or not RAIM is available. The following section discusses the determination of suitable alarm limits. RAIM performance will also be influenced by the constellation design, the probability of system failures and the UERE budgets for each satellite. RAIM analysis is clearly dependent on these key parameters, which must be set appropriately for the navigation system and the specific application.

These summary figures give an overall indicator of RAIM performance over time and space. Figures 1 and 2 show the percentage of time that RAIM is available at the minimum probability level for each sample point over 24 hours, using a vertical alarm limit of $18 \mathrm{~m}$. With 288 samples per point (every 5 minutes) one sample represents $0.35 \%$, therefore figures have not been categorised beyond one decimal place. If RAIM is available at each 5-minute sample over a 24hour period it is likely that the availability at that point would be $100 \%$ regardless of recording rate.

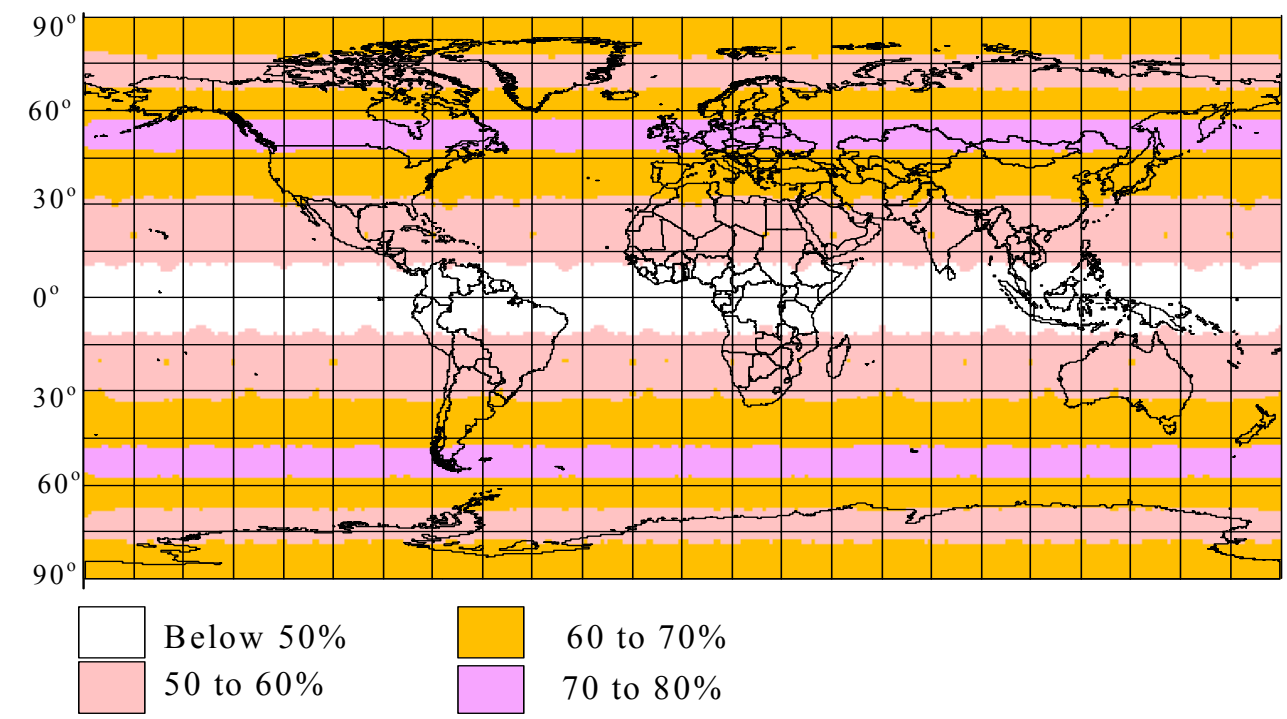

Figure 1: RAIM Availability - Galileo Only 


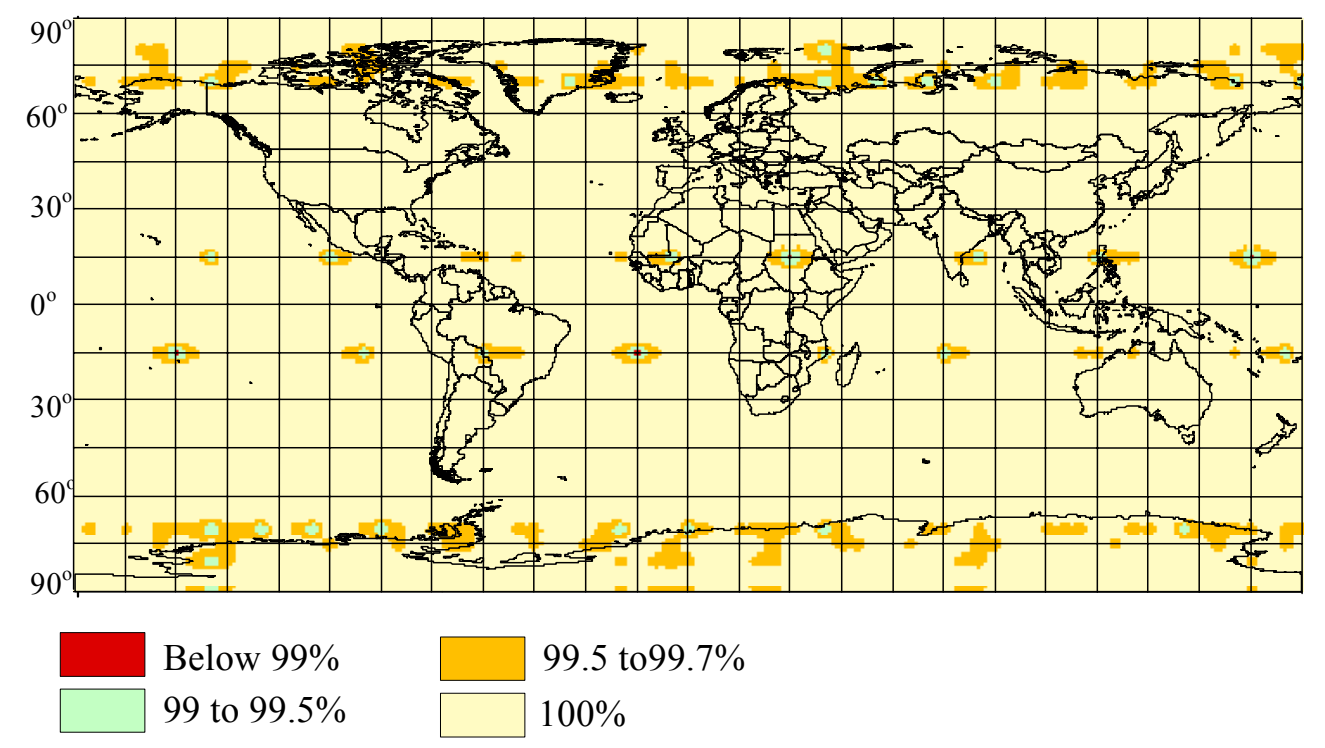

Figure 2: RAIM Availability - Galileo + GPS

The global distribution plots again emphasise that RAIM is available for a far greater proportion of the time, nearing $100 \%$, when a combined Galileo and GPS constellation is used. These plots also show that RAIM performance varies with latitude - availability is greatest at mid latitudes, with the poorest performance being found near the equator. There are also more localised patterns that may need to be studied in greater detail to optimise the constellation design for specific coverage areas.

RAIM performance and availability has been shown to be a function of a number of factors: -

(1) The number of redundant observations and improved constellation geometry in the outlier detection algorithm.

(2) The probability with which an error must be detected.

(3) The size of acceptable error.

(4) The quality of the observations used. 


\section{Determination of Horizontal and Vertical Alarm Limits}

The results presented have been determined based on the horizontal and vertical alarm limits as specified for the Galileo system in May 2000. The simulation tools developed for this study have also been used to determine realistic horizontal and vertical alarm limits given set probability levels. In practice, alarm limits need to be specified for a particular positioning/navigation application and should not be dictated by the capabilities of one particular navigation system, nevertheless, these figures provide some useful guides to attainable performance.

Preliminary analyses have been carried out using the two probability levels described in Table 3 , to determine the position shifts resulting from the smallest gross error that can be detected. Shifts larger than the computed value will be detected, but it is not possible to detect any smaller shifts. The results of this analysis for regional coverage, based on the scenarios in Table 3, are shown in Figures 3 to 6 .

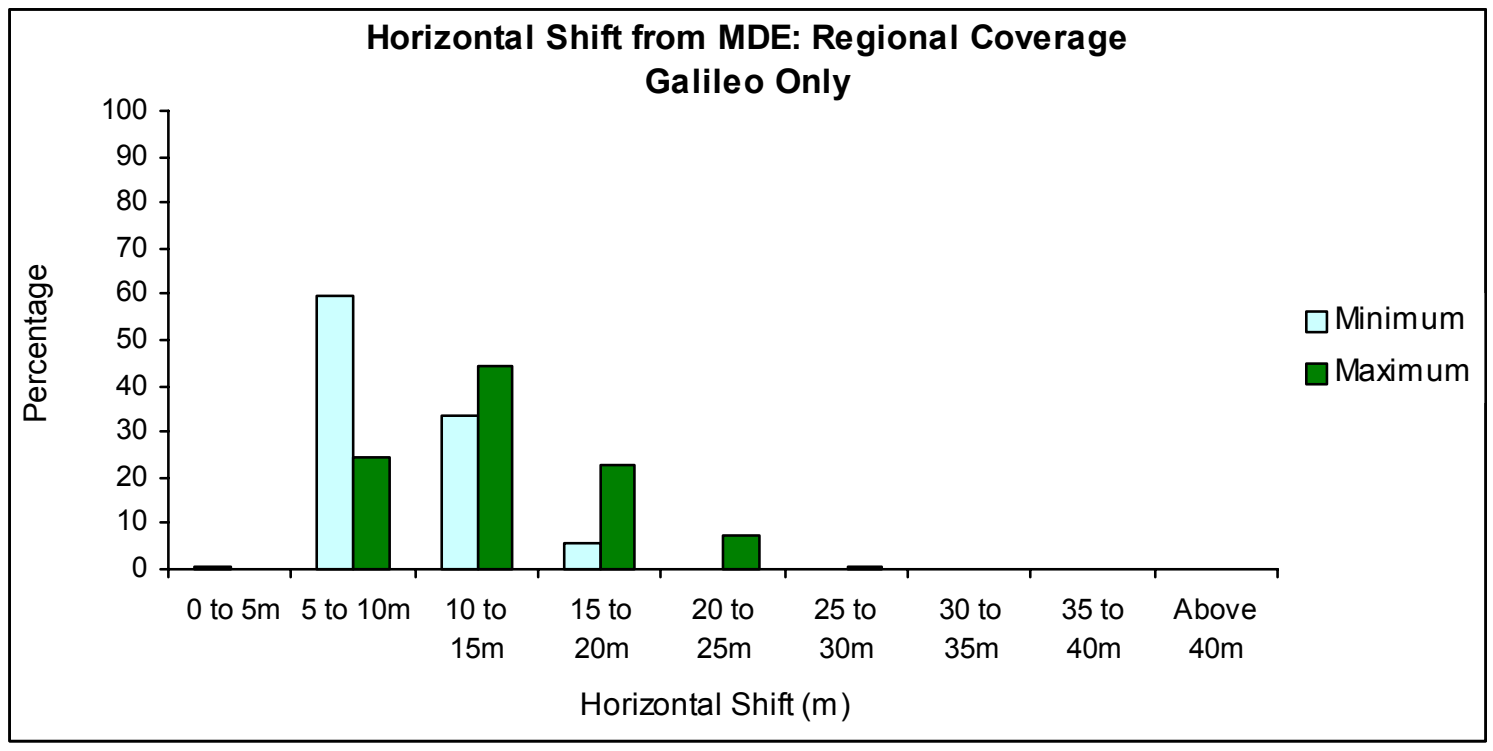

Figure 3: Horizontal Shifts with Minimum and Maximum Probability Levels - Galileo Only 


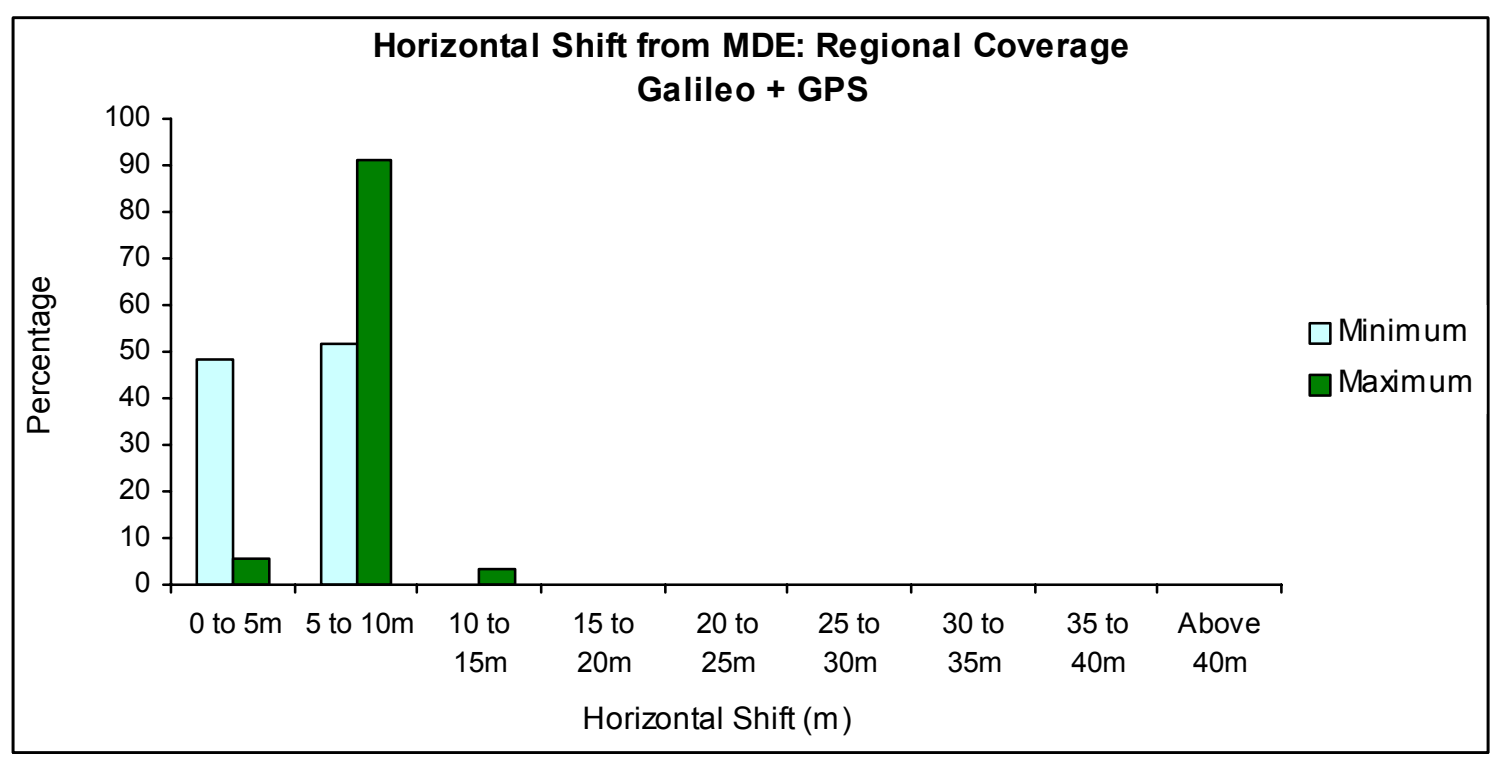

Figure 4: Horizontal Shifts with Minimum and Maximum Probability Levels - Galileo + GPS

The horizontal position shift resulting from the MDE at the minimum probability level is below $20 \mathrm{~m}$ for $99.95 \%$ of the time using Galileo alone. Applying the maximum probability levels this figure falls to $92 \%$. Depending on the specified probability levels for missed detection and false alarm, a realistic horizontal alarm limit for the Galileo constellation would be between 25 and 30m. This would allow RAIM availability approaching $100 \%$.

The horizontal position shift resulting from the MDE is below $10 \mathrm{~m}$ for $99.95 \%$ of the time at the minimum probability level, using a Galileo/GPS constellation. Applying the maximum probability levels this figure is $96.88 \%$.

For the same probability level, smaller horizontal shifts can be detected more of the time using a combined Galileo/GPS constellation. Using this constellation the horizontal alarm limit could be reduced significantly, certainly to less than $15 \mathrm{~m}$, whilst still allowing close to $100 \%$ RAIM availability. 


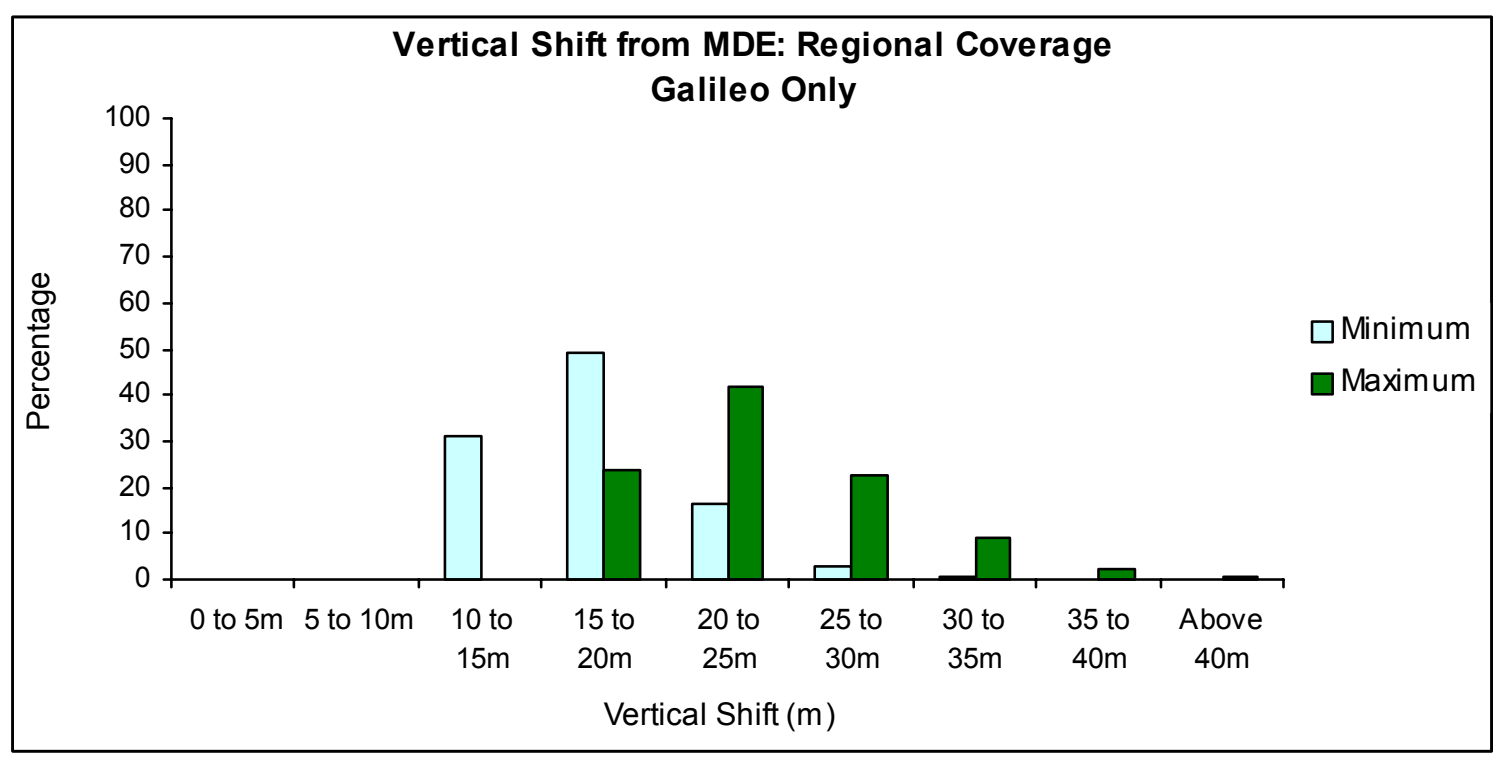

Figure 5: Vertical Shifts with Minimum and Maximum Probability Levels - Galileo Only

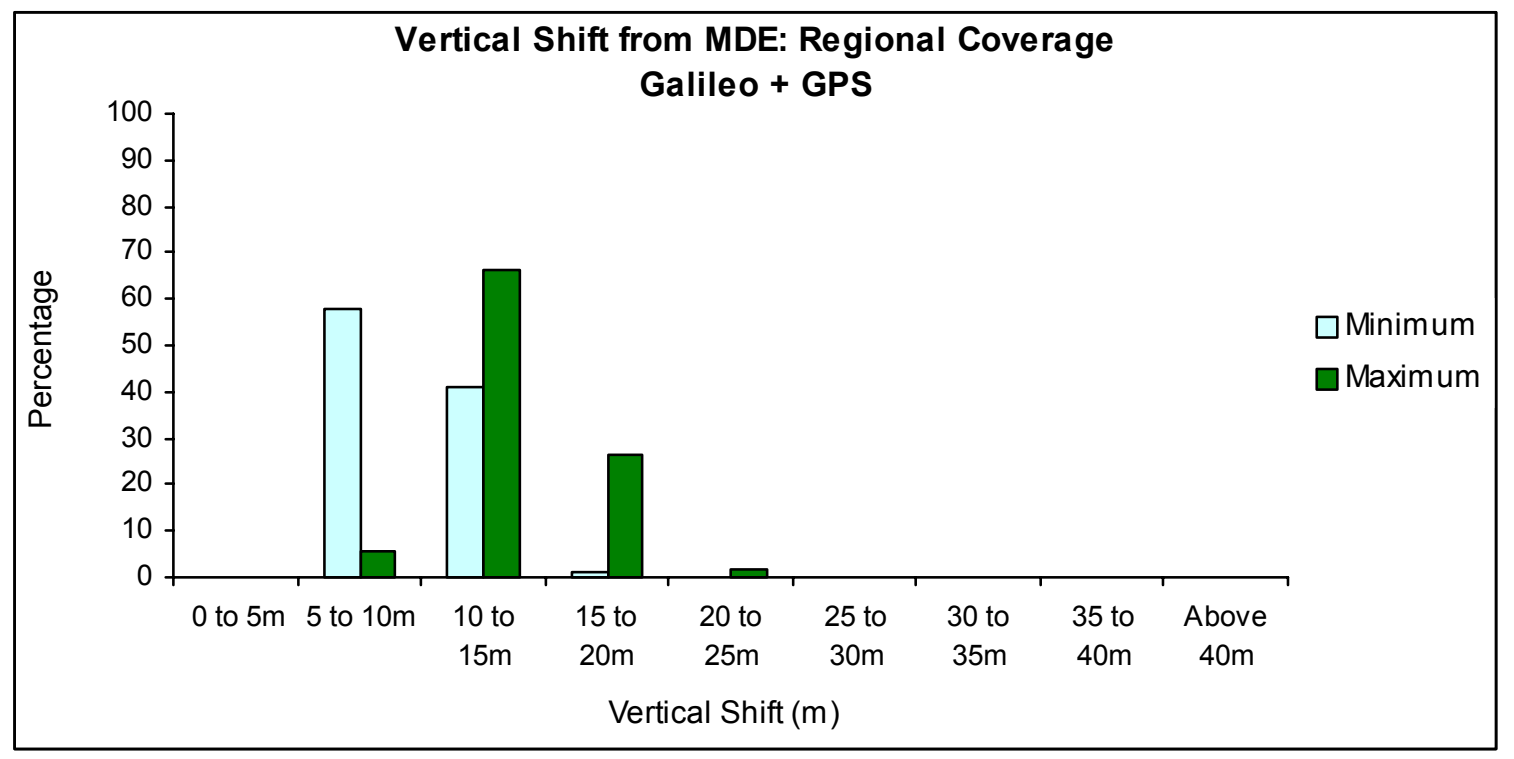

Figure 6: Vertical Shifts with Minimum and Maximum Probability Levels - Galileo + GPS

The vertical position shift resulting from the MDE at the minimum probability level is below $30 \mathrm{~m}$ 97.2\% of the time using Galileo alone. Applying the maximum probability levels this figure falls to $89.0 \%$. Depending on the specified probability levels for missed detection and false alarm, a realistic vertical alarm limit for the Galileo constellation would be between 30 and $40 \mathrm{~m}$. This would allow RAIM availability approaching 100\%. 
With the minimum probability level, the vertical shift from the MDE is below $15 \mathrm{~m} 98.4 \%$ of the time using a Galileo/GPS constellation. At the maximum probability level, the vertical shift from the MDE is below $15 \mathrm{~m}$ only $69.8 \%$ of the time.

For the same probability level, smaller vertical shifts can be detected more of the time using a combined Galileo/GPS constellation. Using this constellation the vertical alarm limit could be reduced significantly, probably to between 20 and $25 \mathrm{~m}$, whilst still allowing close to $100 \%$ RAIM availability.

\section{CONCLUSIONS}

The results presented demonstrate the performance improvement, in terms of RAIM availability, when GPS is used to complement Galileo. The use of a combined constellation can either reduce the minimum position shift that can be detected at a given probability level, or, can increase the probability with which a blunder of a given size will be detected. Overall, there is a significant improvement in integrity using a combined Galileo/GPS system compared to the performance using the Galileo system alone.

Using a vertical alarm limit of $18 \mathrm{~m}$, RAIM availability using Galileo alone is well below the levels required for many navigation applications, even when relatively relaxed values for the probability of missed detection and false alarm are used. Analysis has shown that an achievable vertical alarm limit using the proposed Galileo system would be between 30 and $40 \mathrm{~m}$.

This study has also emphasised the need to establish some key parameters before conducting a comprehensive assessment of a navigation system's integrity performance. These include the performance thresholds for an application, specified in terms of alarm limits, integrity risk, timeto-alarm, false alarm rates and probability of missed detection. Also, the other design parameters of the navigation system must be clearly defined, this includes information regarding the 
constellation configuration and corresponding User Equivalent Range Error (UERE) budgets, and the probability of a satellite failure.

Performance improvements from using a combined constellation must be related to the requirements of particular navigation services in order to judge their significance. At the time this research was carried out, the navigation service requirements were under review and the Galileo system architecture had not been fully defined. As these elements become increasingly well defined, further work should be undertaken to test the system performance with these service requirements in mind, and to assess the benefits that using a combined Galileo/GPS constellation could bring to particular users.

\section{DISCLAIMER}

The authors are solely responsible for the publication and it does not represent the opinion of the European Community. The Community is not responsible for the use that might be made of the data appearing in the publication.

\section{REFERENCES}

Conley, R. (1998). Results of the GPS JPO's GPS Performance Baseline Analysis: The GOSPAR Project. NAVIGATION, Journal of the Institute of Navigation, Vol.45, No.1, Spring, 31-38.

Corrigan, T.M., Hartranft, J.F., Levy, L.J., Parker, K.E, Pritchett, J.E., Pue, A.J., Pullen, S., \& Thompson, T. (1999). GPS Risk Assessment Study Report. The John Hopkins University, VS-99007, January.

Cross, P.A., (1983). Advanced Least Squares Applied to Position Fixing. Working Paper No.6, North East London Polytechnic, United Kingdom. 
Fernow, J.P., \& Loh, R. (1994). Integrity Monitoring in a GPS Wide-Area Augmentation System (WAAS). Proceedings of the Third International Conference on Differential Satellite Navigation Systems, DSNS 94, April 18-22, London, 1-8.

Lannelongue, S., Le Buan, C., Mimoun, D., Watson, T., \& Scarda, S. (2000). Performance Budget File. Galileo Overall Architecture Definition, Alcatel Space, Toulouse, France.

Ochieng, W.Y., \& Cross, P.A. (2000a). Analysis of the GPS Mordernisation Process (31 pp.). Task 1 Final Report Submitted to ASPI, Toulouse, France, June.

Ochieng, W.Y., \& Cross, P.A. (2000b). Performance Potential of a Combined Galileo/GPS Navigation System (71 pp.). Tasks $2 \& 3$ Final Report submitted to ASPI, Toulouse, France, July.

Ochieng, W.Y., Shardlow, P.J., \& Johnston, G. (1999). Advanced Transport Telematics Positioning Requirements: An Assessment of GPS Performance in Greater London. The Journal of Navigation. Volume 52, Number 3, September, 342-355.

Phlong, W.S., \& Elrod. B.D. (1994). Availability Characteristics of GPS and Augmentation Alternatives. NAVIGATION - Journal of the Institute of Navigation, Vol.40, No.4 , 409-428.

RTCA (1998). The Minimum Operational Performance Standards (MOPS) for Global Positioning System/Wide Area Augmentation System Airborne Equipment. Document No. RTCA/DO-229a. June 8.

RTCA (1991). The Minimum Operational Performance Standards (MOPS) for Airborne Supplemental Navigation Equipment using GPS. Document No. RTCA/DO-208. July 12. 
Turner, D., Shaw, M and Sandhoo, K. (2000). GPS Modernisation. In proceedings of GNSS 2000, Edinburgh, May 4.

Vincent, J.P. (2000). Galileo Architecture Baseline Definition. Alcatel Space, Toulouse, France. 


\section{BIOGRAPHIES}

Washington Ochieng is leader of the Imperial College Engineering Geomatics Group (ICEGG). He holds a PhD degree in Space Geodesy from the University of Nottingham. Before joining Imperial College he was a Senior Post-Doctoral Research Assistant at the Institute of Engineering Surveying and Space Geodesy (IESSG) at the University of Nottingham and Principal Engineer at Racal Electronics Limited. He teaches courses in Engineering Geomatics and Transport Telematics. His main research interest is in the design of space based navigation systems and their application to engineering problems.

Kevin Sheridan is a Research Associate with the Engineering Geomatics Group at Imperial College. Kevin obtained his $\mathrm{PhD}$ degree in Geomatic Engineering from University College London. His research interests include GPS attitude determination, transport telematics and GNSS design.

Mr. Knut Sauer is a PhD student at ICEGG, working primarily in the field of satellite navigation augmentation systems.

Xiao Liang Han is a Senior Research Officer at the Centre for Transport Studies at Imperial College. He has over 15 years experience in statistical analysis and model building.

Paul Cross is the Leica Professor of Geomatic Engineering and Head of the Geomatic Engineering department at University College London. He is responsible for teaching and research related to geodesy, surveying and navigation.

Mr. Stephanne Lannelongue, Ms. Nadia Ammour and Mr. Kris Petit are Systems Engineers with Alcatel Space Industries (ASPI). 


\begin{tabular}{|l|l|l|l|l|l|}
\hline $\begin{array}{l}\text { Elevation } \\
\text { angle } \mathbf{(}^{\mathbf{(}} \text { ) }\end{array}$ & \multicolumn{2}{l|}{$\begin{array}{l}\text { US DoT/DoD } \\
\text { estimates }\end{array}$} & \multicolumn{3}{l|}{ GALA estimates $(\mathbf{m} \boldsymbol{\sigma})$} \\
\cline { 2 - 6 } & $\begin{array}{l}\text { GPS } \\
\text { L1/L2 }\end{array}$ & $\begin{array}{l}\text { GPS } \\
\text { L1/L5 }\end{array}$ & $\begin{array}{l}\text { GPS } \\
\text { L1/L2 }\end{array}$ & $\begin{array}{l}\text { GPS } \\
\text { L1/L5 }\end{array}$ & $\begin{array}{l}\text { Galileo } \\
\text { CAS }\end{array}$ \\
\hline 10 & 2.35 & 2.18 & 5.23 & 3.65 & 4.37 \\
\hline 30 & 2.07 & 1.88 & 2.77 & 1.93 & 2.02 \\
\hline 50 & 2.06 & 1.86 & 2.28 & 1.60 & 1.58 \\
\hline 70 & 2.05 & 1.85 & 2.25 & 1.57 & 1.52 \\
\hline 90 & 2.05 & 1.85 & 2.27 & 1.58 & 1.52 \\
\hline
\end{tabular}

Table 1: UERE Budgets for GPS and Galileo Satellites

\begin{tabular}{|l|l|}
\hline Integrity Risk & $10^{-7} /$ hour \\
\hline Horizontal Alarm Limit & $18 \mathrm{~m}$ \\
\hline Vertical Alarm Limit & $18 \mathrm{~m}$ \\
\hline Time-To-Alarm & $6 \mathrm{~s}$ \\
\hline MASKING ANGLE $\mathbf{(}^{\mathbf{0}}$ ) & 5 \\
\hline
\end{tabular}

Table 2: Galileo Navigation Performance Requirements

\begin{tabular}{|l|l|l|l|}
\hline & $\beta$ & $\alpha$ & $\boldsymbol{\delta}_{\boldsymbol{i}}$ \\
\hline Scenario 1 & 0.999 & 0.9998 & 6.86 \\
\hline Scenario 2 & 0.999996 & 0.999999 & 9.50 \\
\hline
\end{tabular}

Table 3: Probabilities of Missed Detection and False Alarm

\begin{tabular}{|l|l|l|l|l|}
\hline \multirow{2}{*}{} & \multicolumn{2}{|c|}{$\begin{array}{c}\text { Regional } \\
\left.\mathbf{7 5}^{\mathbf{0}} \mathbf{S} \text { to } \mathbf{7 5}^{\mathbf{0}} \mathbf{N}\right)\end{array}$} & \multicolumn{2}{c|}{ Global } \\
\cline { 2 - 5 } & $\begin{array}{l}\text { Galileo } \\
\text { Only }\end{array}$ & $\begin{array}{l}\text { Galileo } \\
\text { +GPS }\end{array}$ & $\begin{array}{l}\text { Galileo } \\
\text { Only }\end{array}$ & $\begin{array}{l}\text { Galileo } \\
\text { + GPS }\end{array}$ \\
\hline Horizontal & & & & \\
\hline $\begin{array}{l}\text { Minimum } \\
\text { Probability }\end{array}$ & $99.18 \%$ & $100 \%$ & $99.27 \%$ & $100 \%$ \\
\hline $\begin{array}{l}\text { Maximum } \\
\text { Probability }\end{array}$ & $85.14 \%$ & $100 \%$ & $86.05 \%$ & $100 \%$ \\
\hline Vertical & & & & $99.97 \%$ \\
\hline $\begin{array}{l}\text { Minimum } \\
\text { Probability }\end{array}$ & $65.05 \%$ & $99.97 \%$ & $65.38 \%$ & $99.97 \%$ \\
\hline $\begin{array}{l}\text { Maximum } \\
\text { Probability }\end{array}$ & $6.40 \%$ & $93.11 \%$ & $7.28 \%$ & $91.73 \%$ \\
\hline
\end{tabular}

Table 4: RAIM Availability Statistics with Alternative Probability Levels 